\title{
Trends in use and cost of initial cancer treatment in Ontario: a population-based descriptive study
}

\author{
Claire de Oliveira PhD, Karen E. Bremner BSc, Reka Pataky MSc, Nadia Gunraj MPH, Mahbubul Haq MSc, \\ Kelvin Chan MD MSc, Winson Y. Cheung MD MPH, Jeffrey S. Hoch PhD, Stuart Peacock DPhil,
} Murray D. Krahn MD MSc

\section{Abstract}

Background: Cancer incidence and treatment-related costs are rising in Canada. We estimated health care use and costs in the first year after diagnosis for patients with 7 common types of cancer in Ontario to examine temporal trends in patterns of care and costs.

Methods: We selected patients aged 19-44 years who had received a diagnosis of melanoma, breast cancer (female only), testicular cancer or thyroid cancer, in addition to patients aged 45 years and older who had received a diagnosis of breast (female only), prostate, lung or colorectal cancer, between 1997 and 2007. Patients were identified from the Ontario Cancer Registry. Using linked administrative databases, we determined use and costs of chemotherapy, radiotherapy, cancer-related surgery, other admissions to hospital and home care. We adjusted all costs to 2009 Canadian dollars.

Results: We identified 20821 patients aged 19-44 years and 178797 patients aged 45 years and older. The greatest increases in costs during the study period were for melanoma, breast cancer, colorectal cancer, lung cancer and prostate cancer $(p<0.05)$. For prostate and lung cancers, mean costs increased 50\% (from $\$ 11490$ and $\$ 22037$ to $\$ 15170$ and $\$ 34473$, respectively). Mean costs doubled for breast (from \$15 460 and \$12 909 to \$35 977 and \$29 362 for younger and older patients, respectively) and colorectal cancers (from \$24 769 to $\$ 43964$ ), and nearly tripled for melanoma (from $\$ 3581$ to $\$ 8934$ ). Costs related to hospital admissions accounted for the largest portion of total costs. The use of chemotherapy, radiotherapy and home care generally increased for all cancers.

Interpretation: The significant increase in mean costs of initial cancer treatment among the patients included in this study was primarily due to more patients receiving adjuvant therapy and home care, and to the increasing expenditures for these services and cancer-related surgeries. Understanding trends in health care use and costs can help policy-makers to take the necessary measures to achieve a more accountable, high-performing health care system.

1 he incidence of cancer in Canada has increased recently owing to population growth and aging. ${ }^{1}$ This increased incidence, in addition to the increased use of expensive new drugs and technologies to treat cancer, has led to a rise in cancer-related expenditures, which consume a growing share of limited health care budgets. ${ }^{2-4}$

Previous studies have shown that many cancer-related costs are incurred in the year after diagnosis..$^{5-7}$ However, which resources and health services contribute most to the overall cost and whether rising prices or increased use can explain increased expenditures is not yet fully understood.

We examined temporal trends in use of health care resources and costs for melanoma, breast cancer (in women only), testicular cancer and thyroid cancer among patients aged 19-44 years, and for breast (in women only), prostate, lung and colorectal cancers among patients aged 45 years and older to understand how patterns of care and associated costs for these cancers have changed. Our age cut-offs are based on previous work because there is no standard international definition of "young adult"; our rationale is that young adulthood stretches from the end of adolescence to the start of menopause, in which the latter is known to induce marked changes in the cancer profile of women. ${ }^{8}$ We chose these types of cancer because they account for about $60 \%$ of all cancers in each of the age groups included in our study. We sought to identify current cancer care needs to help predict trends in cancer care use and expenditures.

Competing interests: None declared.

This article has been peer reviewed.

Correspondence to: Claire de Oliveira, claire.deoliveira@ camh.ca CMAJ Open 2013.DOI:10.9778/cmajo.20130041 


\section{Methods}

\section{Cohort selection}

We selected patients from the Ontario Cancer Registry, the population-based registry for Canada's largest province (population 13.2 million). ${ }^{9}$ We included patients with cancer aged 19 years and older whose first diagnosis occurred between Jan. 1, 1997, and Dec. 31, 2007, and who lived for at least 12 months after receiving their diagnosis. We included patients assigned a single, valid International Classification of Diseases for Oncology, third edition (ICD-O-3), topography code for a primary cancer (melanoma, or breast, thyroid or testicular cancer for patients aged 19-44 years, and breast, prostate, lung or colorectal cancer for patients aged 45 years and older) with no second cancer diagnosed within 90 days of the initial diagnosis (Appendix 1 at www.cmajopen.ca/content/1/4/E151/suppl/DC1). The 20 most frequent histology codes for each cancer were reviewed by two practising oncologists (K.C. and W.C) to exclude patients with missing, unusual or incorrect tumour types.

\section{Defining resource categories and costs}

We estimated and compared trends in use and costs for all health care services (e.g., inpatient and outpatient hospital care, physician services, outpatient drugs, diagnostic tests, home care, continuing care and long-term care) provided during the 12-month period after diagnosis (including the date of diagnosis). In addition, we examined the trends in use and mean costs for specific treatments: cancer-related surgery, chemotherapy, radiotherapy and other cancer-related admissions to hospital, as well as home care, given its growing importance in Ontario in caring for people with cancer.

We obtained data on radiotherapy and data on chemotherapy drugs provided under the New Drug Funding Program from Cancer Care Ontario. We accessed administrative data- bases (i.e., the Discharge Abstract Database, National Ambulatory Care Reporting System, Continuing Care Reporting System, Ontario Health Insurance Plan [OHIP] Claims History Database, Ontario Drug Benefit Program, Ontario Home Care Administrative System and Home Care Database) through the Institute for Clinical Evaluative Sciences in Toronto, Ontario (Table 1). Individual patient-level data were linked using OHIP numbers and anonymized for analyses.

We employed validated costing methods used in previous work to obtain robust estimates of use and costs (Appendix 2, at www.cmajopen.ca/content/1/4/E151/suppl/DC1). ${ }^{10}$ To select all cancer-related surgeries during a patient's stay in hospital, we used the Canadian Classification of Diagnostic, Therapeutic, and Surgical Procedures codes (up to and including Mar. 31, 2002) and the Canadian Classification of Health Interventions codes (from Apr. 1, 2002, onward). ${ }^{11}$ Because these codes are not comparable, we were not able to reliably estimate cancer-related surgeries and other admissions to hospital for 2002. Cost estimates for other resources were available in the data, or we obtained them from other sources (previously published work, community care access centres and the Ontario Ministry of Health and Long-term Care). ${ }^{12,13}$

We adjusted costs to 2009 Canadian dollars using the health care component of the Statistics Canada Consumer Price Index for Ontario. ${ }^{14}$

\section{Statistical analysis}

We determined the percentage of patients receiving each treatment or modality of care, the respective mean cost of care in each year of diagnosis and the total mean cost of care for each form of cancer by year of diagnosis.

We used linear regression models, with percentage of patients receiving the treatment and cost as the dependent variables and year of diagnosis as the independent variable to

Table 1: Data captured from administrative databases

\begin{tabular}{|ll|}
\hline Database* & \multicolumn{1}{c|}{ Data } \\
\hline New Drug Funding Program & Chemotherapy drugs \\
\hline Activity Level Reporting System & Radiotherapy \\
\hline $\begin{array}{l}\text { Ontario Health Insurance Plan Claims } \\
\text { History Database }\end{array}$ & $\begin{array}{l}\text { Diagnostic tests, physician services, chemotherapy visits, } \\
\text { emergency department visits before 2002 }\end{array}$ \\
\hline Ontario Drug Benefit plan data & $\begin{array}{l}\text { Outpatient prescription drugs for patients aged } \geq 65 \text { yr, oral } \\
\text { antineoplastic drugs, long-term care indicator }\end{array}$ \\
\hline CIHI-Discharge Abstract Database & $\begin{array}{l}\text { Inpatient admissions to hospital (cancer-related surgeries and } \\
\text { other admissions), same-day surgeries before 2002 }\end{array}$ \\
\hline $\begin{array}{l}\text { CIHI-National Ambulatory Care } \\
\text { Reporting System }\end{array}$ & $\begin{array}{l}\text { Emergency department visits after 2002; same-day surgeries } \\
\text { after 2002 }\end{array}$ \\
\hline Continuing Care Reporting System & Stays in complex continuing care facilities \\
\hline $\begin{array}{l}\text { Ontario Home Care Administrative } \\
\text { System }\end{array}$ & \begin{tabular}{l} 
Home care up to Mar. 31, 2005 \\
\hline Home Care Database
\end{tabular} \\
\hline $\begin{array}{l}\text { Note: CIHI = Canadian Institute for Health Information. } \\
\text { Funding Program and Activity Level Reporting System, which were available through Cancer Care Ontario. }\end{array}$ \\
\hline
\end{tabular}


determine the significance of trends over time. For each slope that we estimated, we calculated the 2 -sided $p$ values and $95 \%$ confidence intervals (CIs) through normal approximation. ${ }^{15,16}$

This study was approved by the Research Ethics Board at the University of Toronto, Toronto, Ontario.

\section{Results}

Our cohorts included 20821 patients aged 19-44 years and 178797 patients aged 45 years and older (of whom 101426 were aged 65 years and older). The most common cancer among patients in the younger group was breast cancer in women (43.9\%), followed by thyroid cancer (25.9\%) (Table 2). Prostate and breast cancers (36.4\% and $31.1 \%$, respectively) were the most common cancers among patients in the older age group (Table 2). Patients in the younger age group were predominantly female $(76.3 \%)$, whereas patients in the older group were predominantly male $(54.0 \%)$ (Table 2 ).

We found a significant increase in mean costs during the first year after diagnosis for melanoma, and for breast, lung, colorectal and prostate cancers from 1997 to 2007 ( $p<0.05$ ) (Figure 1). Among younger patients, mean costs associated with breast cancer more than doubled (from \$15 460 in 1997 to $\$ 35977$ in 2007); costs associated with melanoma nearly tripled (from \$3581 in 1997 to $\$ 8934$ in 2007). For older patients, mean costs almost doubled for breast and colorectal cancers (from \$12 909 and \$24 769 in 1997 to \$29 362 and $\$ 43964$ in 2007, respectively), and increased by about $50 \%$ for prostate and lung cancers (from \$11 490 and \$22 037 in 1997 to $\$ 15170$ and $\$ 34471$ in 2007, respectively). Colorectal and lung cancers presented the largest mean total costs throughout our analysis period. Overall, the greatest changes worth noting in first-year postdiagnosis costs occurred for breast (women in both age groups), colorectal and lung cancers.

\section{Breast cancer}

Chemotherapy use rose from $70.1 \%$ of patients to $78.1 \%$ among those aged $19-44$ years $(p>0.05)$, and from $26.7 \%$ of patients to $43.9 \%(p<0.05)$ among those 45 years of age and older (Figure 2; Appendix 3, at www.cmajopen.ca/content /1/4/E151/suppl/DC1). Chemotherapy costs increased 5-fold for all patients (from $\$ 2286$ to $\$ 11834$ for patients aged 1944 years, $p<0.05$; from $\$ 791$ to $\$ 5978$ for patients aged 45 years and older, $p<0.05$ ) (Appendix 3 ).

The percentage of patients with breast cancer in the younger group undergoing radiotherapy increased from $49.4 \%$ to $70.8 \%$ during the study period $(p<0.05)$ (Figure 3$)$, and corresponding expenditures tripled (from $\$ 2067$ in 1997 to $\$ 6268$ in 2007) $(p<$ 0.05; annual increase \$393, 95\% CI \$224-\$563) (Appendix 3). The proportion of patients aged 45 years and older receiving radiotherapy increased from $43.5 \%$ to $66.3 \%$ during the study period $(p<0.05)$ (Figure 3 ), and the corresponding costs more than tripled, from $\$ 1620$ to $\$ 4920(p<0.05)$ (annual increase \$297, 95\% CI \$155-\$438) (Appendix 3).

The proportion of patients using home care increased from $64.0 \%$ to $80.0 \%(p<0.05)$ among those aged $19-44$ years and from $59.6 \%$ to $72.2 \%(p<0.05)$ among those 45 years of age and older (Figure 4); the corresponding costs increased for both age groups (Appendix 3).

The proportion of patients undergoing cancer-related surgery remained constant (about 94\%, Figure 5A). Corresponding costs for patients aged 19-44 years decreased from 1997 to $2001(p<0.05)$ and increased from 2003 to 2007 ( $p<$ 0.05 ) (Figure $5 \mathrm{~B})$. Costs for patients aged 45 years and older were similar in terms of both value and trend. The proportion of patients requiring other admissions to hospital also remained relatively constant throughout the study period, as did the associated costs (Appendix 3).

\section{Colorectal cancer}

Among patients with colorectal cancer, the proportion of those using home care increased from $51.8 \%$ to $63.1 \%$ during the study period $(p<0.05)$ (Figure 4$)$. The corresponding costs followed a similar pattern, increasing from $\$ 2190$ to $\$ 3679$ ( $p<0.05$ ) (Appendix 3). Although few patients received radiotherapy, its use and cost increased slightly over the course of the study (Figure 3 and Appendix 3).

About $90 \%$ of patients had surgery during the study period, and the associated costs increased significantly (Figure

Table 2: Patient characteristics, by age group

\begin{tabular}{|c|c|c|}
\hline \multirow[b]{2}{*}{ Characteristic } & \multicolumn{2}{|c|}{ Patients, no. $(\%)^{*}$} \\
\hline & $\begin{array}{c}\text { Age }<45 \mathrm{yr} \\
n=20821\end{array}$ & $\begin{array}{l}\text { Age } \geq 45 \mathrm{yr} \\
n=178797\end{array}$ \\
\hline \multicolumn{3}{|l|}{ Type of cancer } \\
\hline Breast (women only) & 9147 (43.9) & $55670(31.1)$ \\
\hline Thyroid & 5394 (25.9) & - \\
\hline Melanoma & 3816 (18.3) & - \\
\hline Testis & $2464(11.8)$ & - \\
\hline Lung & - & $14372 \quad(8.0)$ \\
\hline Colorectal & - & $43621(24.4)$ \\
\hline Prostate & - & $65134(36.4)$ \\
\hline \multicolumn{3}{|l|}{ Age at diagnosis, yr } \\
\hline Mean & 36.5 & 66.2 \\
\hline Median & 38.0 & 66.0 \\
\hline Standard deviation & 6.2 & 10.5 \\
\hline \multicolumn{3}{|l|}{ Sex } \\
\hline Female & $15882(76.3)$ & $82266(46.0)$ \\
\hline Male & $4939(23.7)$ & $96531(54.0)$ \\
\hline \multicolumn{3}{|c|}{ Neighbourhood income quintile } \\
\hline Data missing & $10 \quad(0.5)$ & $712 \quad(0.4)$ \\
\hline 1 (lowest) & $3520(16.9)$ & $31330(17.5)$ \\
\hline 2 & $3917(18.8)$ & $35712(20.1)$ \\
\hline 3 & 4224 (20.3) & $35308(19.7)$ \\
\hline 4 & $4428(21.3)$ & $36006(20.1)$ \\
\hline 5 (highest) & $4627(22.2)$ & $39729(22.2)$ \\
\hline Rural residence & 2202 (10.6) & 27274 (15.3) \\
\hline
\end{tabular}


5). The proportion of patients who required other admissions to hospital remained constant (about 78\% before 2002 and $85 \%$ after 2002). The corresponding costs for these admissions increased with time (Appendix 3).

The proportion of patients receiving chemotherapy showed only a modest increase, whereas the corresponding costs almost doubled (Figure 2).

\section{Lung cancer}

The proportion of patients receiving chemotherapy for lung cancer doubled during the study period, from $18.1 \%$ to $37.5 \%(p<0.05)$ (Figure 2), and the corresponding costs more than tripled, from $\$ 685$ to $\$ 2576(p<0.05)$ (Appendix 3). The proportion of patients receiving radiotherapy increased from $23.7 \%$ to $32.2 \%$ ), with corresponding mean costs more than doubling, from $\$ 676$ to $\$ 1498$ (Figure 3 and Appendix 3). The proportion of patients using home care increased from $42.4 \%$ to $52.3 \%(p<0.05)$ (Figure 4$)$, with corresponding mean costs increasing from $\$ 1784$ to $\$ 2236(p<0.05)$ (Appendix 3).

The proportion of patients who underwent surgery decreased from $79.1 \%$ in 1997 to $74.7 \%$ in 2001 , and increased from $67.7 \%$ in 2003 to $72.8 \%$ in $2007(p<0.05)$ (Figure 5A). Associated mean costs were relatively constant from 1997 to 2001 (about $\$ 9000$ ), but increased from $\$ 8697$ in 2003 to $\$ 10737$ in $2007(p<0.05)$ (Figure 5B). The proportion of patients requiring other admissions to hospital decreased from $60.0 \%$ in 1997 to $56.8 \%$ in 2001 and remained relatively constant (at about 58\%) from 2003 to 2007; the corresponding mean costs were constant before 2002 but increased from \$3489 in 2003 to $\$ 4472$ in 2007 (Appendix 3).

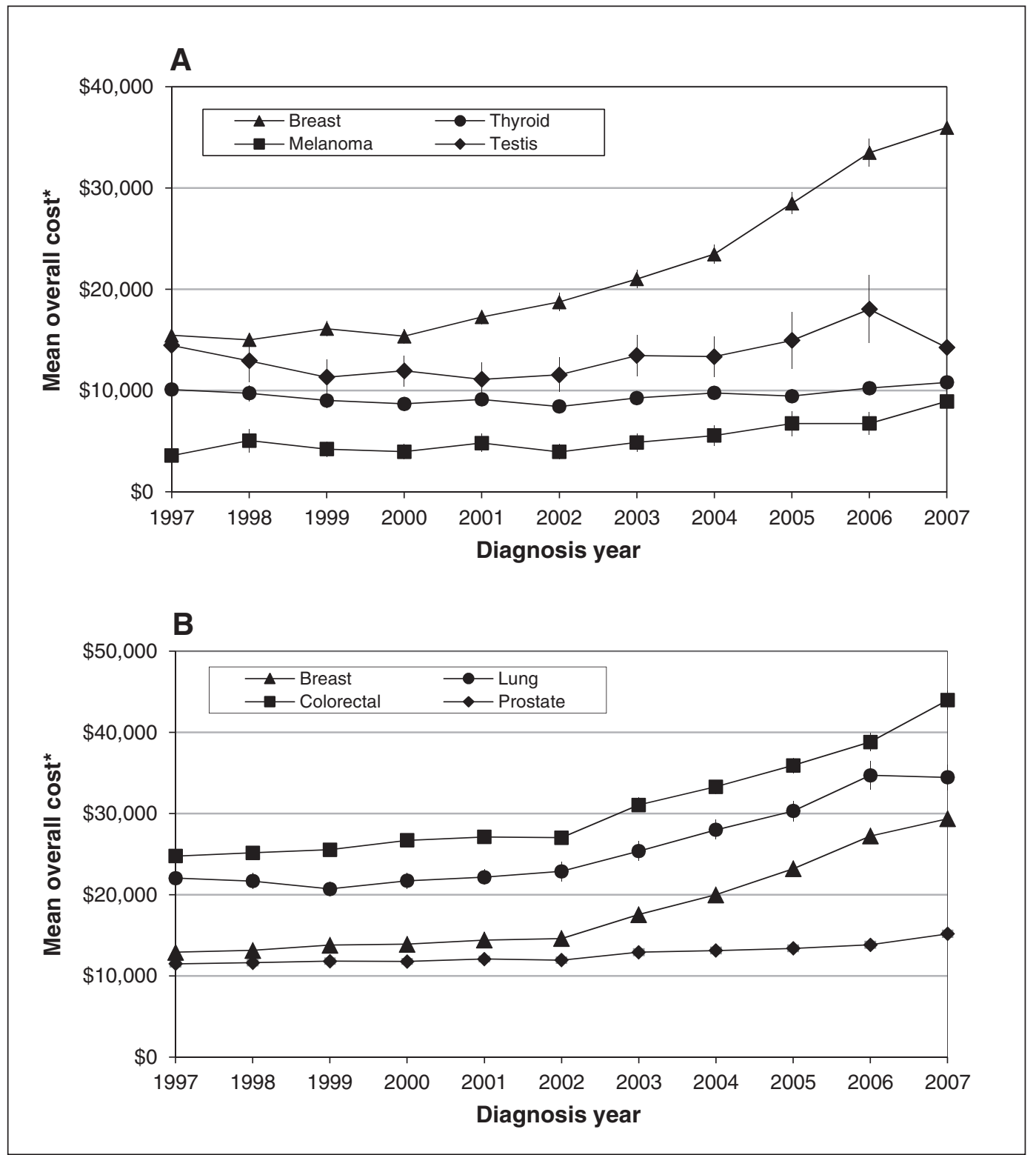

Figure 1: Trends in mean cost during the first year after diagnosis for $(\mathrm{A})$ patients less than 45 years of age and (B) patients 45 years of age and older. Error bars indicate $95 \%$ confidence intervals. *Values in 2009 Canadian dollars. 


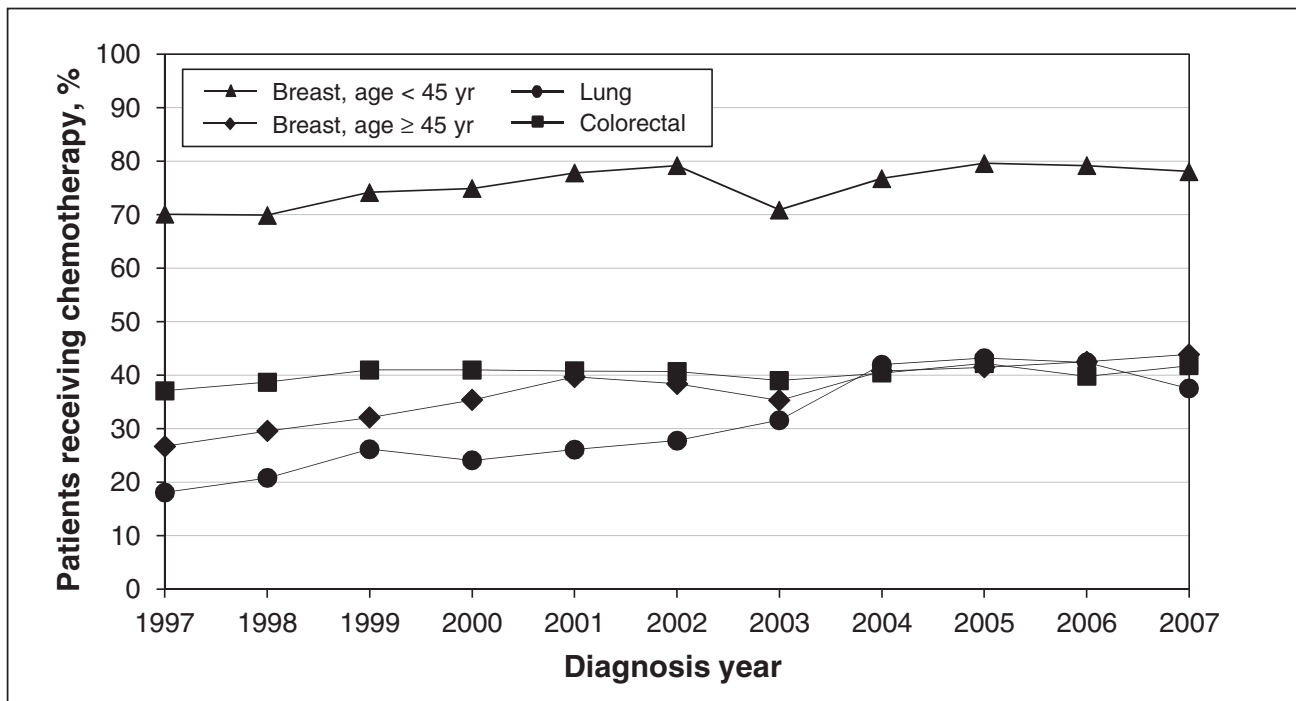

Figure 2: Trends in the percentage of patients receiving chemotherapy during the first year of treatment.

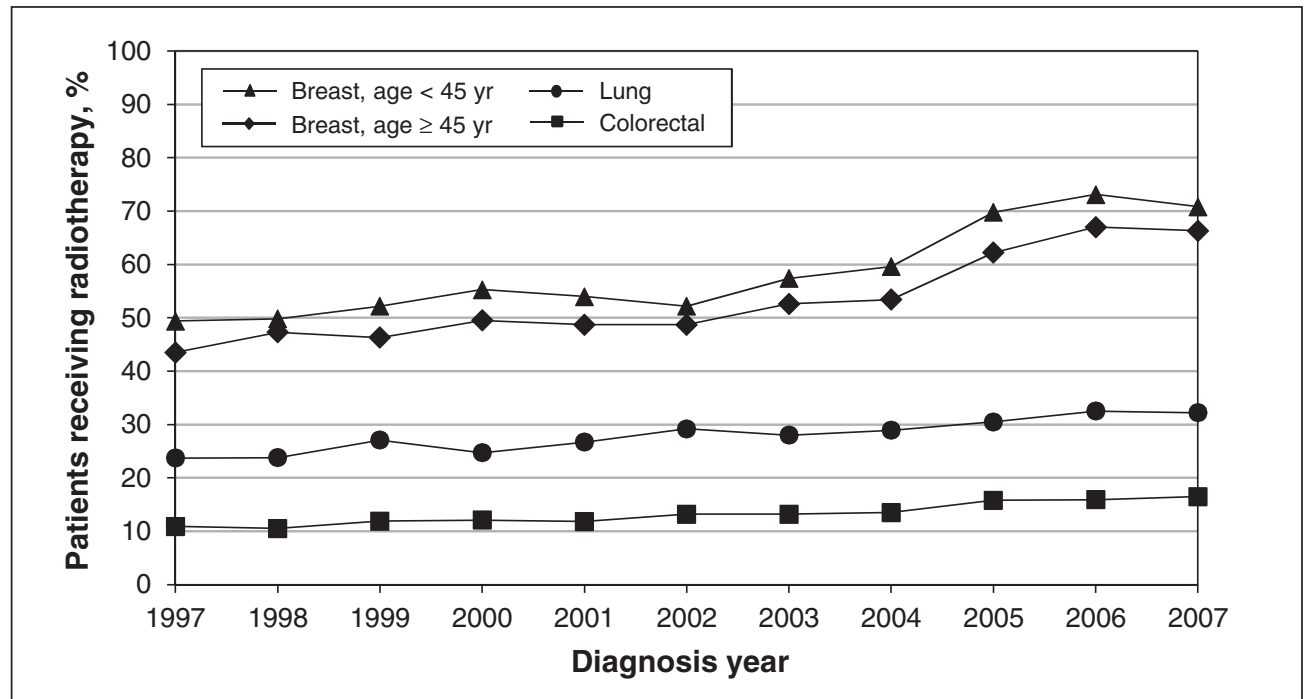

Figure 3: Trends in the percentage of patients receiving radiotherapy during the first year of treatment.

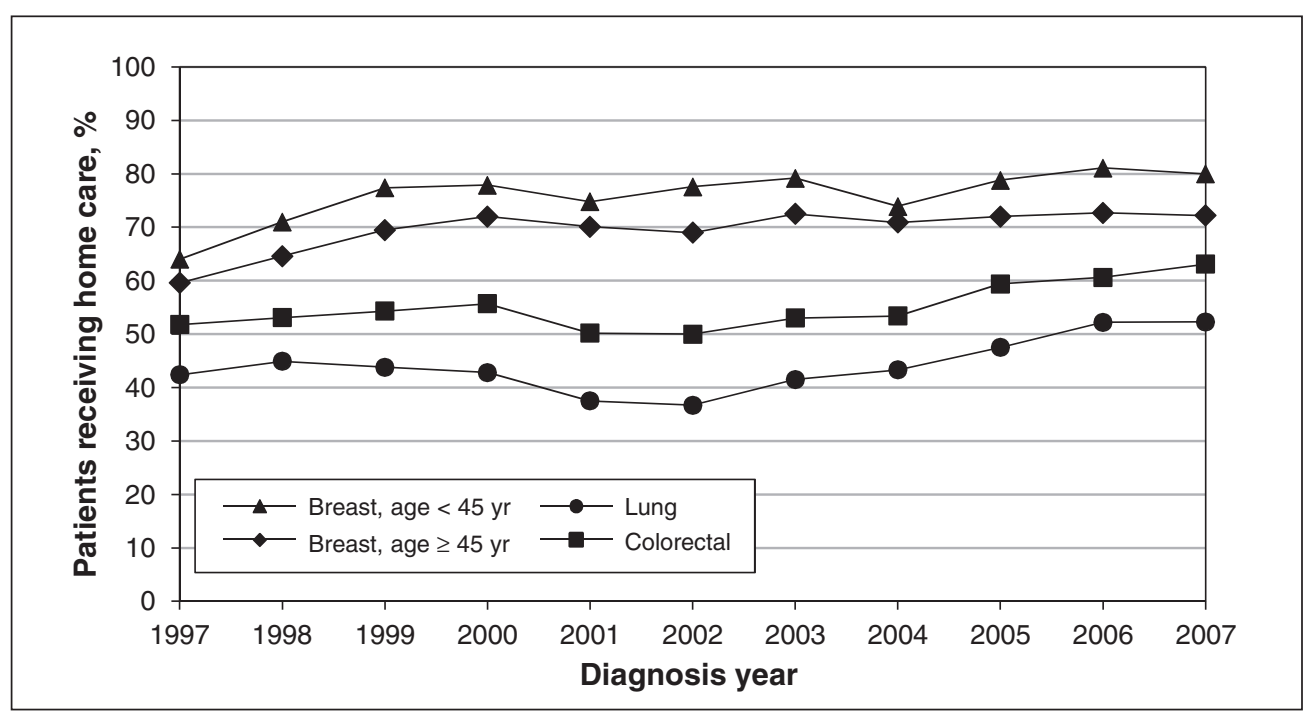

Figure 4: Trends in the percentage of patients receiving home care during the first year of treatment. 


\section{OPEN}

\section{Research}

\section{Interpretation}

We found significant increases in mean total costs for breast, prostate, lung and colorectal cancers in Ontario from 1997 to 2007 among patients who lived for at least 1 year after receiving their diagnosis. The mean costs associated with breast cancer more than doubled for patients aged 19-44 years and doubled for patients aged 45 years and older. Among patients in the older age group, the mean costs associated with colorectal cancer doubled, and the costs associated for prostate and lung cancer increased by about $50 \%$.

Increases in the costs associated with treatment were partially due to increased use of chemotherapy and radiotherapy. These results are concordant with those from studies in the United States. ${ }^{7,17}$ Higher use of home care and rising costs for cancer-related surgeries also contributed to this upward trend. Patterns of care and practice tend to be similar among Canadian provinces and other developed countries $;{ }^{17}$ thus, our results are likely extendable Canada-wide and mirror patterns of care elsewhere. Although these increases are not specific to cancer care ${ }^{18}$ cancer is one of the diseases with the greatest economic burdens in Canada. ${ }^{19}$

Chemotherapy use increased over time, particularly among younger patients with breast cancer and older patients with lung cancer. This increase could reflect a rise in patients receiving adjuvant chemotherapy, which has been clinically proven to improve patient survival..$^{20-22}$ The use of treatments with personalized medicine and biologic agents, which are often more expensive than traditional chemotherapy agents, has also increased, ${ }^{23}$ which may help further explain this trend.

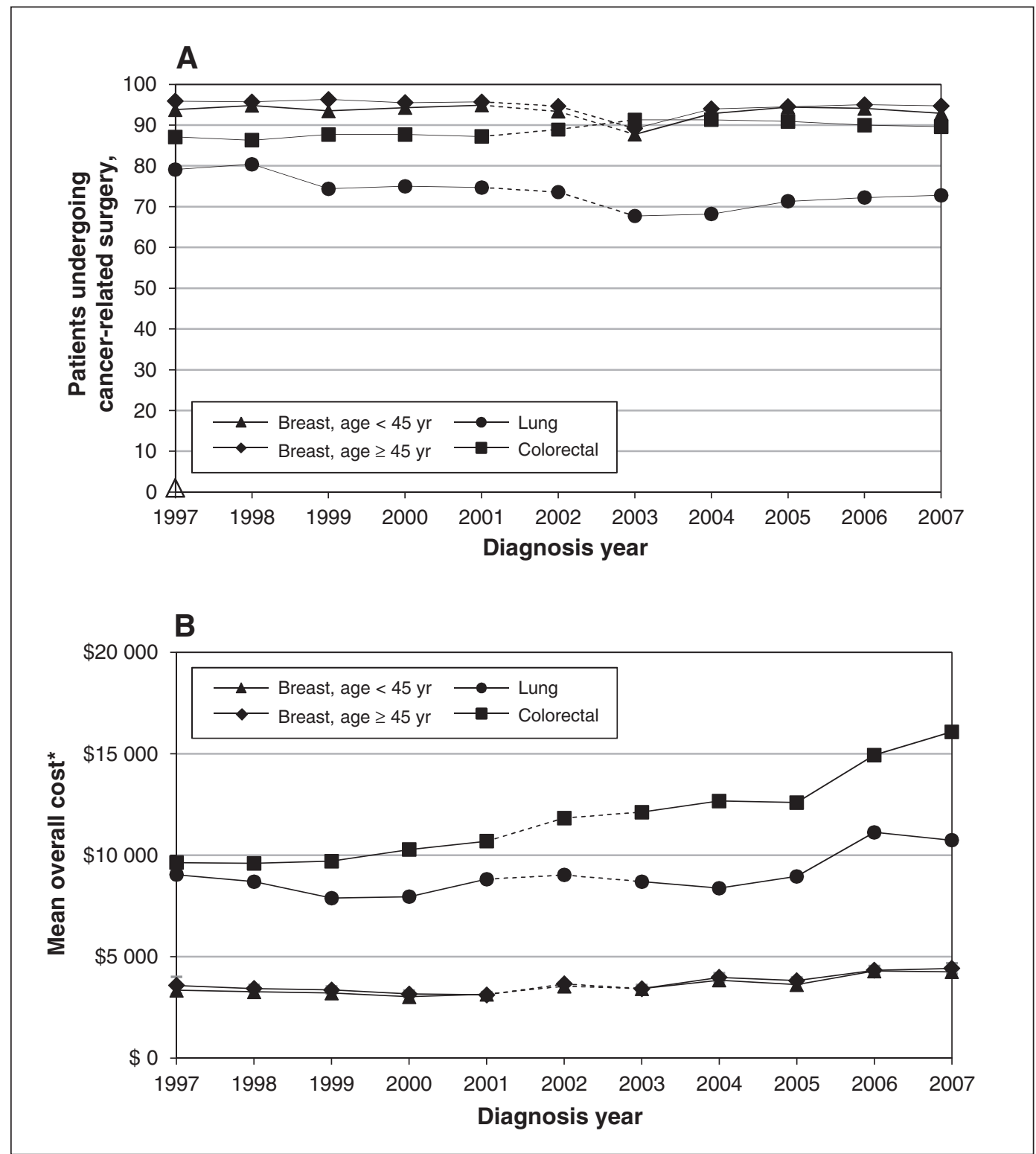

Figure 5: Trends in (A) the percentage of patients undergoing cancer-related surgeries and $(B)$ the mean cost of surgery during the first year of treatment. *Values in 2009 Canadian dollars. 
The increase in the use and cost of radiotherapy, particularly for breast cancer, is probably due to increased availability, changes in patterns of care and more sophisticated methods, such as conformal and intensity-modulated radiotherapy. For example, oncologists may use adjuvant radiotherapy in patients who undergo lumpectomy, a procedure that became more common during the study period..$^{24}$

Admissions to hospital for cancer-related surgeries and other reasons accounted for most of the costs associated with the initial phase of care. For most cancers, the proportion of patients undergoing surgery remained relatively constant over time, although the trend for women with breast cancer may have been slightly different had we included same-day procedures, such as lumpectomies. Surgery-related costs generally increased, particularly for breast, colorectal and lung cancers, suggesting an increase in price, likely because of the use of more advanced and expensive surgical techniques, such as laparoscopic and robotic surgeries.

The increase we saw in the use of home care, especially among older patients, is not exclusive to cancer. ${ }^{25}$ Postacute home care is typically used for patients with cancer in the first 30 days after they are discharged from hospital. ${ }^{13}$ The increased use of home care is a trend that the Ontario government seems to be adopting, ${ }^{26}$ with calls to expand coverage across Canada. ${ }^{27}$

Little research has been done on trends in cancer care and their associated costs. Warren and colleagues ${ }^{7}$ reported significant increases in the costs of initial care for patients aged 65 years and older for breast, lung and colorectal cancers from 1991 to 2002 in the United States, with lung and colorectal cancers having the highest overall costs. The authors also found that admissions to hospital accounted for the largest portion of the overall cost, and radiotherapy use generally increased. Our findings are similar, but our cost estimates for each treatment category are lower, possibly owing to differences in analysis periods (their initial phase of care included 2 months before and 12 months after diagnosis), unit costs, patient age and, in some cases, patterns of care. When we restricted our sample to patients 65 years of age and older, our results were qualitatively the same, with slightly higher estimates of use and costs than we determined in our overall results.

\section{Strengths and limitations}

Our study included patients less than 65 years of age, an age group that is not often included in the literature. In addition, our study compared many different cancer sites, thus providing direct comparability of their relative costs. Finally, we used administrative data, detailed costing methods and a large population-based sample of all adults in Ontario. Our study is one of the few to use such data to examine trends in cancerrelated expenditures.

We identified several limitations to our study. We examined only the most prevalent cancer sites for the two age groups and select cancer-related resources. A change in procedural-related coding systems limited our data such that we could not examine admissions to hospital that occurred in 2002. Our chemotherapy estimates were based on OHIP physician claims and may underestimate actual use, and our cost of radiotherapy is based on a dated estimate. In addition, the Ontario Drug Benefit program only covers patients 65 years of age and older or patients with special circumstances; thus, our cost estimate of prescription drugs is likely an underestimate. Finally, we did not look beyond the first year postdiagnosis and may have included costs of the predeath period for some patients, which may have biased our cost estimates upwards. This is likely to be of concern for cancers with short survival (i.e., colorectal and lung cancers).

\section{Conclusion}

We found significant increases in the costs of initial treatment for most of the types of cancer we examined for patients in both age groups during our study period. The greatest changes in costs occurred for breast, colorectal and lung cancers, primarily because more patients received adjuvant therapy and home care and because of the rising costs for these services and for cancer-related surgeries. It is important to determine the drivers behind this increase as costs continue to rise. Chemotherapy will likely place a strain on Ontario's health care budget; however, costs could be reduced in some ways. For example, personalized medicine and the use of gene expression profiling among women with breast cancer have the potential to be cost-saving. ${ }^{28,29}$

Understanding trends in use and costs is particularly important for planning and resource allocation. Our robust estimates of trends in cancer-related expenditures can help policy- and decision-makers, and serve as important inputs for researchers interested in modelling cost implications of emerging technologies and practices in Canada and similar countries.

\section{References}

1. Canadian Cancer Society's Steering Committee on Cancer Statistics. Canadian Cancer Statistics 2012. Toronto (ON): Canadian Cancer Society; 2012.

2. Constant A, Benda MC, Ariste R, et al. Is the price of cancer treatment falling? Ottawa (ON): Health Canada, Applied Research and Analysis Directorate, Health Policy Branch Working Paper; 2006.

3. Health Canada. Progress report on cancer control in Canada. Ottawa (ON): Health Canada; 2004

4. National Cancer Institute of Canada. Canadian Cancer Statistics 2004. Toronto $(\mathrm{ON})$ : Canadian Cancer Society, National Cancer Institute of Canada, Statistics Canada, Provincial/Territorial Cancer Registries, Health Canada; 2004.

5. Brown ML, Riley GF, Schussler N, et al. Estimating health care costs related to cancer treatment from SEER-Medicare data. Med Care 2002;40(8 Suppl): IV-104-17.

6. Warren JL, Brown ML, Fay MP, et al. Costs of treatment for elderly women with early-stage breast cancer in fee-for-service settings. 7 Clin Oncol 2002;20: 307-16.

7. Warren JL, Yabroff KR, Meekins A, et al. Evaluation of trends in the cost of initial cancer treatment. 7 Natl Cancer Inst 2008;100:888-97.

8. Cancer Care Ontario. Cancer in young adults in Canada. Toronto (ON): Cancer Care Ontario, Public Health Agency of Canada, Canadian Cancer Society; 2006.

9. Hall S, Schulze K, Groome P, et al. Using cancer registry data for survival studies: the example of the Ontario Cancer Registry. 7 Clin Epidemiol 2006;59:67-76.

10. de Oliveira C, Bremner K, Pataky R, et al. Understanding the costs of cancer care before and after diagnosis for the 21 most common cancers in Ontario: a population-based descriptive study. CMA7 Open 2013;1:E1-8.

11. Canadian Institute for Health Information. Coding standards for version 2009 ICD-10-CA and CCI, revised September 2009. Ottawa (ON): The Institute; 2009.

12. Earle C, Coyle D, Smith A, et al. The cost of radiotherapy at an Ontario regional cancer centre: a reevaluation. Crit Rev Oncol Hematol 1999;32:87-93.

13. Wodchis WP, Bushmeneva K, Nikitovic M, et al. Guidelines on person-level costing using administrative databases in Ontario. Toronto (ON): Health System Performance Research Network; 2011.

14. The Consumer Price Index. Ottawa (ON): Statistics Canada; 2012. Cat no 62-001-X. 
15. Brown ML, Riley GF, Potosky AL, et al. Obtaining long-term disease specific costs of care: application to Medicare enrollees diagnosed with colorectal cancer. Med Care 1999;37:1249-59.

16. Yabroff KR, Lamont EB, Mariotto A, et al. Cost of care for elderly cancer patients in the United States. 7 Natl Cancer Inst 2008;100:630-41.

17. Lang K, Marciniak MD, Faries D, et al. Trends and predictors of first-line chemotherapy use among elderly patients with advanced non-small cell lung cancer in the United States. Lung Cancer 2009;63:264-70.

18. Thorpe KE. The rise in health care spending and what to do about it. Health Aff (Millwood) 2005;24:1436-45

19. Health Canada. Economic burden of illness in Canada. Ottawa (ON): Health Canada; 1998.

20. Palmieri C, Jones A. The 2011 EBCTCG polychemotherapy overview. Lancet 2012;379:390-2.

21. André T, Corrado B, Mounedji-Boudiaf L, et al.; for the Multicenter International Study of Oxaliplatin/5-Fluorouracil/Leucovorin in the Adjuvant Treatment of Colon Cancer (MOSAIC) Investigators. Oxaliplatin, fluorouracil, and leucovorin as adjuvant treatment for colon cancer. NEngl 7 Med 2004;350:2343-51.

22. Kirkwood JM, Ibrahim JG, Sosman JA, et al. High-dose interferon alfa-2b significantly prolongs relapse-free and overall survival compared with the GM2-KLH/QS-21 vaccine in patients with resected stage IIB-III melanoma: results of intergroup trial E1694/S9512/C509801. F Clin Oncol 2001;19:2370-80.

23. Canadian Partnership Against Cancer. The future of cancer control in Canada. A discussion paper on pan-Canadian cancer control priorities for 2012-2017. Toronto (ON): The Partnership; 2011.

24. Fisher B, Anderson S, Bryant J, et al. Twenty-year follow-up of a randomized trial comparing total mastectomy, lumpectomy, and lympectomy plus irradiation for the treatment of invasive breast cancer. NEngl F Med 2002;347:1233-41.

25. Ontario Home Care Association. Home care is the future - supporting seniors to remain at home. Hamilton $(\mathrm{ON})$ : The Association; 2011. Available: www.homecare ontario.ca/public/docs/publications/position\%20papers/2011/supporting-seniors -to-be-at-home.pdf (accessed 2013 Sept. 30).

26. Ontario Ministry of Health and Long-term Care. Ontario helping more seniors live at home longer [news release]. 2012 May 24. Available: http://news.ontario .ca/mohltc/en/2012/05/ontario-helping-more-seniors-live-at-home-longer.html (accessed 2012 Nov. 29)

27. Canadian Healthcare Association. Home care in Canada: from the margins to the mainstream. Ottawa (ON): The Association; 2009.

28. Aspinall MG, Hamermesh RG. Realizing the promise of personalized medicine. Harv Bus Rev 2007;85:108-17.

29. Armstrong K. Can genomics bend the cost curve? FAMA 2012;307:1031-2.

Affiliations: From the Centre for Addiction and Mental Health (de Oliveira); the Toronto Health Economics and Technology Assessment Collaborative (de Oliveira, Bremner); the University Health Network
(Bremner, Krahn), Toronto, Ont.; the Canadian Centre for Applied Research in Cancer Control (Pataky, Hoch, Peacock); the British Columbia Cancer Agency (Pataky), Vancouver, BC; the Institute for Clinical Evaluative Sciences, Sunnybrook Health Sciences Centre (Gunraj, Haq); the Department of Medicine, University of Toronto, Sunnybrook Health Sciences Centre and Princess Margaret Hospital (Chan), Toronto, Ont.; the British Columbia Cancer Agency (Cheung, Peacock), Vancouver, BC; St. Michael's Hospital, Cancer Care Ontario, Institute of Health Policy, Management and Evaluation (Hoch), University of Toronto, Toronto, Ont.; the School of Population and Public Health (Peacock), University of British Columbia, Vancouver, BC; and the Toronto Health Economics and Technology Assessment Collaborative, Department of Medicine and Faculty of Pharmacy (Krahn), University of Toronto, Toronto, Ont.

Contributors: Claire de Oliveira contributed to the conceptualization and design of the study, analyzed and interpreted the data and drafted the manuscript. Karen Bremner, Reka Pataky, Kelvin Chan, Winson Cheung, Jeffrey Hoch, Stuart Peacock and Murray Krahn contributed to the conceptualization and design of the study, analyzed and interpreted the data, and revised the manuscript for important intellectual content. Nadia Gunraj selected the patient cohort and linked it with administrative health care data housed at the Institute for Clinical and Evaluative Sciences, Toronto, Ont. Nadia Gunraj and Mahbubul Haq analyzed and interpreted the data and revised the manuscript for important intellectual content. All of the authors approved the final version of the manuscript submitted for publication.

Funding: This project was funded by a research grant from the Canadian Cancer Society Research Institute (grant no. 020200). Murray Krahn is supported by the F. Norman Hughes Chair in Pharmacoeconomics at the Faculty of Pharmacy, University of Toronto, Toronto, Ont.

Disclaimer: This study was supported by the Institute for Clinical Evaluative Sciences (ICES), which is funded by an annual grant from the Ontario Ministry of Health and Long-Term Care (MOHLTC). The opinions, results and conclusions reported in this paper are those of the authors and are independent from the funding sources. No endorsement by ICES or the Ontario MOHLTC is intended or should be inferred.

Supplemental information: For reviewer comments and the original submission of this manuscript, please see www.cmajopen.ca/content/1/4 /E151/suppl/DC1 